\title{
Recycling of Plasmalemma in Chick Tectal Growth Cones
}

\author{
T. P. O. Cheng and T. S. Reese \\ Laboratory of Neurobiology, IRP, NINCDS at The Marine Biological Laboratory, National Institutes of Health, Woods Hole, \\ Massachusetts 02543
}

\begin{abstract}
Growth cones from freeze-substituted intact chick optic tectum were analyzed in serial reconstructions of thin-section electron micrographs in order to determine which internal organelles might contribute membrane for plasmalemmal expansion. These growth cones contain numerous stacked and single lumenless membrane-limited disks; the stacks are arrays of single disks interconnected, and possibly organized, by intervening electron-dense cross-links. The single and stacked disks together account for $80 \%$ of the total intracellular membrane in the growth cones. Single disks frequently lie close to and occasionally contact the filopodial plasmalemma; regularly spaced electron-dense cross-links also occur at these juxtapositions between single disks and the plasmalemma. Some of the juxtaposed disk membranes contact the growth cone plasmalemma, and images of some of these contacts appear to indicate fusion of the disk membrane with the plasmalemma. When excised optic tecta are exposed to cationized ferritin for various times, ferritin micelles appear sequentially in coated pits, coated vesicles, smooth vesicles, vacuoles, and then in stacked and single disks, including some of those contacting the plasmalemma. Because the cytoplasmic disks filled only at the longest times after exposure to ferritin, the membrane continuities between the disks and the plasmalemma are thought to be indicative of exocytosis rather than endocytosis. We propose, therefore, that components of the plasma membrane are recycled through the stacks of lumenless disks in the chick tectal growth cones; the disks therefore represent a pool of internal membrane waiting to be added to the growth cone plasmalemma that could be used for filopodial extension or neuritic extension.
\end{abstract}

The outgrowth of nerve cells to reach specific targets requires directed expansion of both the plasmalemmal surface and the cytoskeletal framework. Thus, expansion is typically restricted to the growing tips of neuronal processes (Harrison, 1910; Speidel, 1933; Bray, 1970; Feldman et al., 1981; Pfenninger and Maylie-Pfenninger, 1981); however, under certain conditions, growth also occurs in the shaft of a neuronal process (Bray and Chapman, 1985). Growing tips of nerve cell processes are characterized by a single cone-shaped varicosity with radiating filopodia and numerous membrane-limited organelles (Tennyson, 1970; Kawana et al., 1971; Yamada et al., 1971; Bunge, 1973;

\footnotetext{
Received May 16, 1986; revised Nov. 17, 1986; accepted Nov, 20, 1986.

We thank Mr. John Murphy for his excellent photographic work.

Correspondence should be addressed to Dr. T. P. O. Cheng, Lab. of Neurobiology, NIH at MBL, Woods Hole, MA 02543.

Copyright @ 1987 Socicty for Neuroscience $0270-6474 / 87 / 061752-08 \$ 02.00 / 0$
}

Landis, 1978; Rees and Reese, 1981; Nordlander and Singer, 1982; Cheng and Reese, 1985). In cultured neurons, these growth cones appear to extend and retract filopodia repeatedly (Bray and Chapman, 1985). Most, if not all, of the membrane necessary for filopodial extension is located in or near the growth cone because amputated growing tips continue to extend filopodia (Bray et al., 1978). However, what membrane is used to cxtend and retract filopodia and what membranc is used for the neuritic growth remains unclear. Subplasmalemmal vesicles have been implicated as a source of membrane added to the growing tip or growth cone of dissociated neurons (Feldman et al., 1981; Pfenninger and Maylie-Pfenninger, 1981), but smooth endoplasmic reticulum (SER) has also been proposed to contribute to the formation of new growth cone plasmalemma (Yamada et al., 1971).

Application of cryogenic techniques has yielded a new picture of the types and distribution of membranes in the growth cone. The numerous subplasmalemmal vesicles and plentiful anastomosing SER are apparently artifacts of aqueous chemical fixation (Rees and Reese, 1981; Cheng and Reese, 1985) because they are not found in freeze-substituted growth cones. Stacks of disk-shaped organelles appear instead when freeze-substitution is applied to chick tectal growth cones (Cheng and Reese, 1985). The functional relationship of the disk-shaped organelles to the growth cone plasmalemma is unknown, although these membrane-limited organelles are concentrated next to the terminal filopodium (Cheng and Reese, 1985).

In order to determine whether any of the various kinds of membrane-limited organelles could supply membrane for filopodial expansion or growth cone extension, we further analyzed the ultrastructural features of the multilamellated stacks and other growth cone organelles with an emphasis on their relationship to the filopodial plasmalemma. Then, in order to specify any sequential transformations of the various membrane structures, we exposed optic tecta to cationized ferritin for various lengths of time prior to freezing. Findings from these experiments suggest a cycle of membrane addition to and removal from the growth cone plasmalemma, which is similar to that in mature synapses (Heuser et al., 1979).

\section{Materials and Methods}

Details of specimen preparation and techniques for computer-assisted reconstruction of serial sections are described elsewhere (Cheng and Reese, 1985) and therefore are only briefly recounted here.

Preparation of specimens. Chicken embryos at $61 / 4-6 \frac{1}{2} \mathrm{~d}$ of incubation were placed in minimal essential medium (GIBCO Lab, Grand Island, NY) to remove the overlying vascular tissues and to dissect out the optic tecta. The dissected specimens were quick-frozen approximately 1 min later as described previously (Cheng and Reese, 1985). The quickfrozen samples were freeze-substituted in a mixture of osmium tetroxide $(5 \%)$ and acctonc for $17 \mathrm{hr}$ at $78^{\circ} \mathrm{C}$, warmed to $-40^{\circ} \mathrm{C}$, rinsed with 

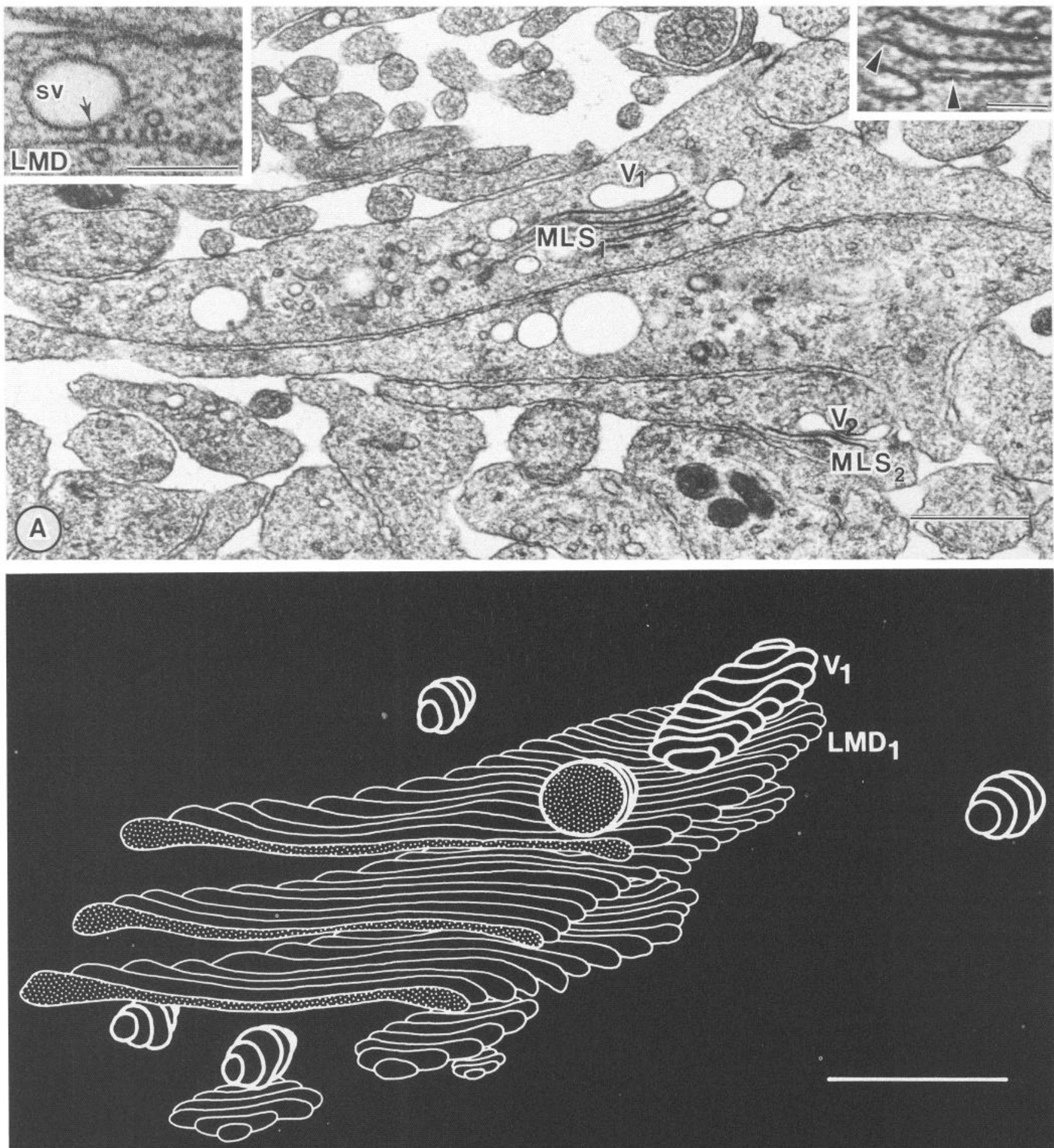

B

Figure 1. Freeze-substituted growth cones $(A)$ from optic tectum and serial reconstruction of one of their multilamellated stacks $(B)$. Vacuoles $\left(V_{1}\right.$ and $V_{2}$ in $\left.B\right)$ are often adjacent to the outermost elements of the multilamellated stacks $\left(M L S_{1}\right.$ and $\left.M L S_{2}\right)$. The lumenless, flat, membranebound stacks frequently terminate in minute lacunae (arrowheads; $A$, right inset). The three-dimensional image $(B)$ was reconstructed from a series of sections through the multilamellated stack, $\mathrm{MLS}_{1}$, shown in $A$; the cut edges of the stack and another organelle are indicated by stippling. In serial reconstructions, the close association of some vacuoles $\left(V_{l}\right)$ with the most proximal lumenless membrane disk $\left(L M D_{l}\right)$ of the stack becomes immediately apparent. The growth cones in $A$ are cut in a plane transverse to the longitudinal axis of the neurite. Projections (arrow; $A$, left inset) typically cross-link disks $(L M D)$ and adjacent smooth vesicles (sv). Scale bars, $0.5 \mu \mathrm{m}$; bars in insets, $0.1 \mu \mathrm{m}$. 

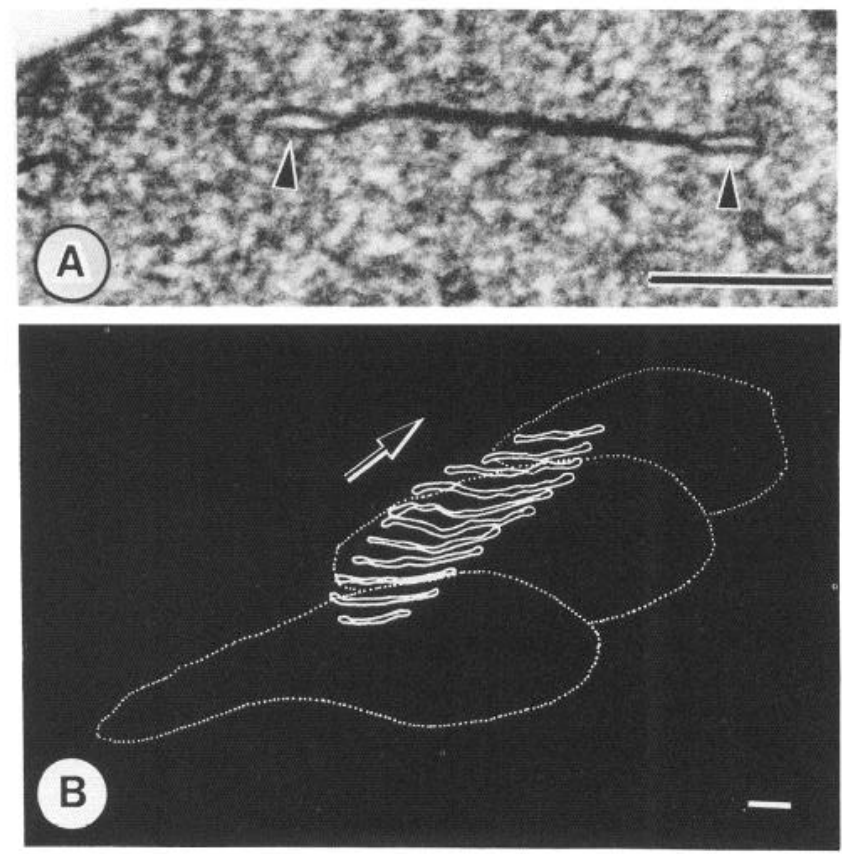

Figure 2. Reconstruction $(B)$ of a series of thin sections through an individual lumenless disk $(A)$. Flat, bilamellated profiles $(A)$ seen in individual sections turn out to be sections through lumenless disks $(B)$. The isolated membrane disks appear to be identical to those in the multilamellated stacks. Again, the flat, lumenless disks typically terminate in minute lacunae (arrowheads, $B$ ). The longitudinal axis of the lumenless disk and the growth cone is indicated by an arrow in $B$. Scale bar, $0.1 \mu \mathrm{m}$.

precooled acetone, and further fixed in another acetone mixture containing acrolein $(5 \%)$ and tannic acid $(0.5 \%)$. Then they were warmed to $0^{\circ} \mathrm{C}$, rinsed with precooled acetone, changed to absolute methanol, and stained with $20 \%$ uranyl acetate in methanol. Finally, they were processed by routine epoxy-embedding techniques for electron microscopy.

Computer-aided reconstruction and quantitation of serial sections. Typically, a ribbon of approximately 250 sections was subdivided into 10 short ribbons and each collected on a Formvar-coated slot grid. After heavy-metal grid staining, the serial sections were examined in a JEOL 200-CX electron microscope at $120 \mathrm{kV}$. During printing of a series, each structure of interest was aligned in best-fit registration with respect to the previous section (Cheng and Reese, 1985). Various features in the aligned serial micrographs (final magnification, 32,500 $\times$ ) were then outlined, using a computer-driven digitizer. After completion of a series, the digitized outlines were built up to construct 3-dimensional images. The software for 3-dimensional reconstruction and measurement of continuous serial sections was written in BASIC. Data acquisition and subsequent graphics display were done with a Tektronix Graphic Tablet 4956 and Graphic System 4054 (Tektronix, Beaverton, OR 97075).

For planimetric measurements, the perimeter of each structure (final magnification, $150,000 \times$ ) was digitized consecutively 7-10 times and the average perimeter (or cross-sectional area) was then multiplied by the section thickness $(90 \mathrm{~nm})$ to give the membrane surface area (or a volume). In order to calibrate the digitizer, plane figures of known perimeters were digitized; the variability between measurements was less than $1.6 \%$.

Cationized ferritin labeling. Two milliliters of sterilized, "cadmiumfree" cationized ferritin solution $(10 \mathrm{mg} / \mathrm{ml}$; cat. no. F7879, Sigma Chemical Co., St Louis, MO) were diluted in $3 \mathrm{ml}$ serum-free incubation medium (GIBCO), and the resulting solution $(4 \mathrm{mg} / \mathrm{ml}$ of ferritin) was used to label freshly excised chick optic tecta. After 15 min incubation at $0^{\circ} \mathrm{C}$, the labeled tecta were warmed to $37^{\circ} \mathrm{C}$ for $5,10,15$, and $30 \mathrm{~min}$ prior to cryogenic fixation. In other experiments, excised tecta were incubated in cationized ferritin medium at $37^{\circ} \mathrm{C}$ for $5,15,30$, and 60 min before being quick-frozen.

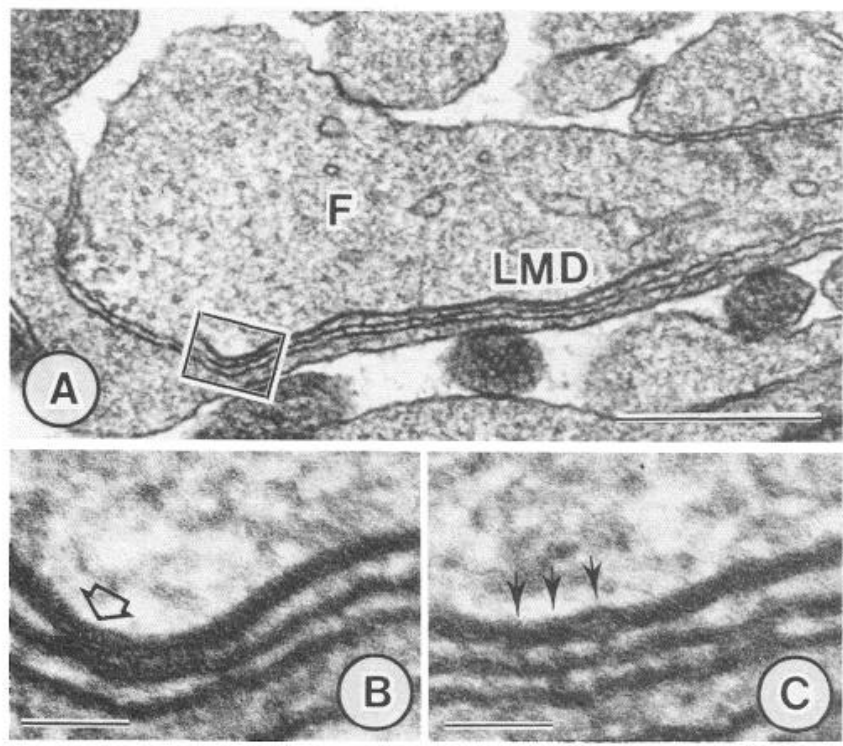

Figure 3. Single lumenless membrane disk $(L M D)$ next to the plasmalemma of a filopodium $(F ; A)$. The area outlined by the rectangle is shown in adjacent sections at higher magnification in $B$ and $C$. This disk is located near the plasmalemma of the filopodium and appears to contact it (open arrow, B). Several filamentous elements (small arrows, $C$ ) cross-bridge the disk and the plasmalemma. Scale bar, in $A$ indicates $0.5 \mu \mathrm{m}$ and those in $B$ and $C$ indicate $0.05 \mu \mathrm{m}$.

\section{Results}

\section{Lumenless membrane disks}

The most prominent structure in freeze-substituted, thin-sectioned growth cones was the multilamellated stack (Fig. 1 $\mathrm{A}$, a novel finding.) Each stack was an array of several (typically $2-$ 5) flat, bilamellated, membrane-limited profiles. It became apparent in the serial reconstructions that each profile was a transverse section of a membrane-limited disk and that these disks packed together to form the multilamellated stack with its longitudinal axis parallel to the longitudinal axis of the growth cone (Fig. $1 B$ ). Most disks lacked a lumen, although minute lacunae were frequent at their ends (Fig. $1 A$, right inset). There was typically one, but occasionally 2 , multilamellated stacks in a growth cone. Groups of single disks (Fig. 2, $A, B$ ) not integrated into a multilamellated stack were frequent inside and at the base of terminal and subterminal filopodia. The single lumenless disks were usually located closer to the plasmalemma than those in the stacks (Fig. 3A); the highest concentrations of lumenless disks were found near the base of the terminal filopodium. The unstacked lumenless disks, unlike those in the multilamellated stacks, did not appear to have any consistent relationship with the various vacuoles described below.

Of the total disk membrane in the growth cones, $68 \%$ was in the distal segment as compared to $1,5,20$, and $6 \%$, respectively, in the terminal neurite, the transition zone, the proximal segment, and the terminal filopodium (successive regions of the growth cone as previously defined by Cheng and Reese, 1985). The single lumenless disks inside and at the base of the terminal and subterminal filopodium were either close to (Fig. 3, $A-C$ ) or contacted (Fig $3 B$ ) the plasmalemma. The contacts between single disks and the plasmalemma appeared to be small, approximately $25 \mathrm{~nm}$ in diameter (Fig. 4). Images of several fortuitous planes of section normal to these contacts suggested that 


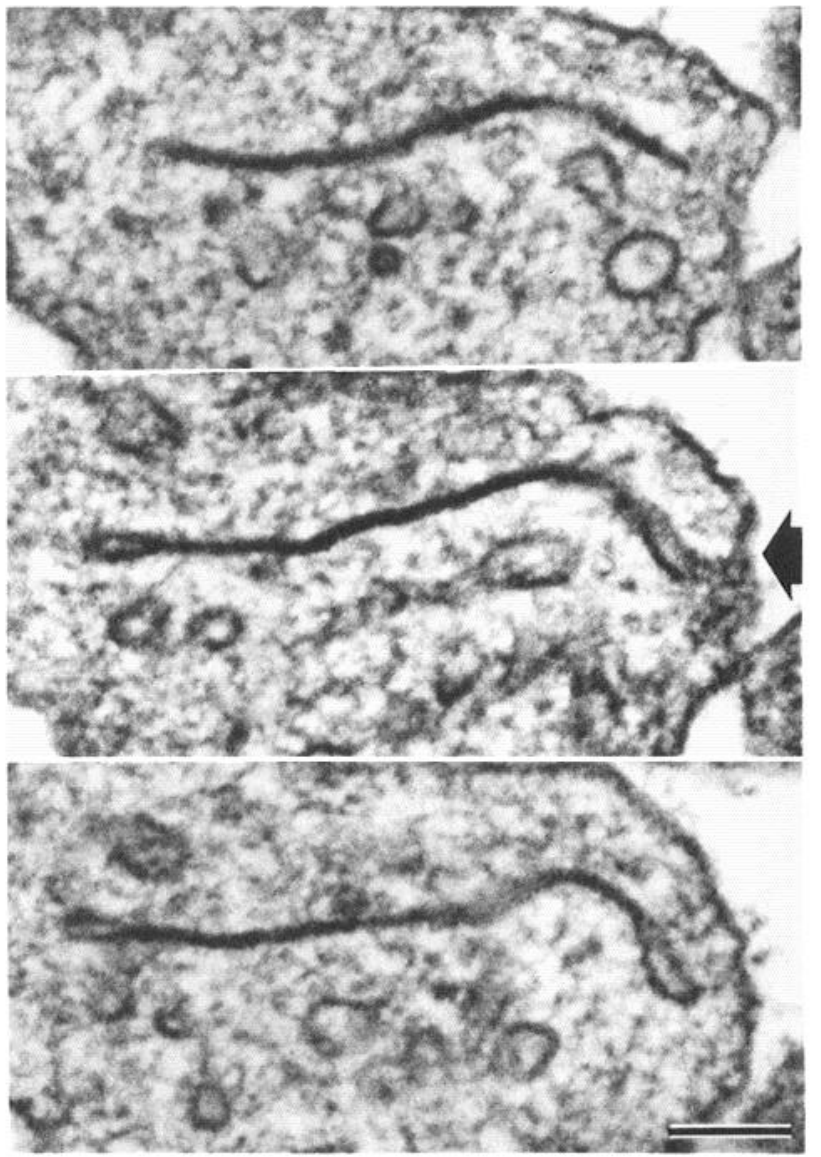

Figure 4. Three contiguous serial sections through a disk that lies near and, in the second section, contacts a filopodial membrane. The disk membrane appears to be continuous with the filopodial membrane within this punctate region of contact (large arrow). Scale bar, $0.1 \mu \mathrm{m}$.

the disk membrane is continuous with the plasmalemma (Fig. 4), as if exocytotic fusion had occurred. Additional evidence for fusion of disks with the plasmalemma came from the sequential appearance of cationized ferritin in these organelles, as described below. Typically $3-5$ fine densities bridged the narrowest region of the gap between a lumenless disk and the adjacent plasmalemma (Fig. 3C). These electron-dense cross-links, as well as those among stacked disks and between vacuoles and disks, were regularly spaced $35-40 \mathrm{~nm}$ apart.

The area of plasma membrane contained in a single disk accounts for $0.87 \mu \mathrm{m}^{2}$ of membrane in the disk illustrated in Figure $2 B$, and the amount contained in the multilamellated stacks is $3.2 \mu \mathrm{m}^{2}$ of membrane in the 3 disks shown in Figure $1 B$. The total quantity of single and stacked disk membrane measured in the tectal growth cones was $26 \mu \mathrm{m}^{2}$, which is approximately $80 \%$ of all the intracellular membrane (multilamellated stacks, single disks, vesicles, and vacuoles). This amount of disk membrane $\left(26 \mu \mathrm{m}^{2}\right)$ also represents $166 \%$ of the surface area of a typical terminal filopodium and is enough membrane to generate 2 filopodial processes $7.9 \mu \mathrm{m}$ long with an average diameter of $0.5 \mu \mathrm{m}$ (the average diameter and length of a terminal filopodium were 0.51 and $6.48 \mu \mathrm{m}$, respectively).

\section{Endosome-like vesicles and vacuoles}

Vesicles (diameter $<150 \mathrm{~nm}$ ) and vacuoles (diameter $>150$ nm; Fig. $1 A$ ) were the most numerous structures in the freeze-
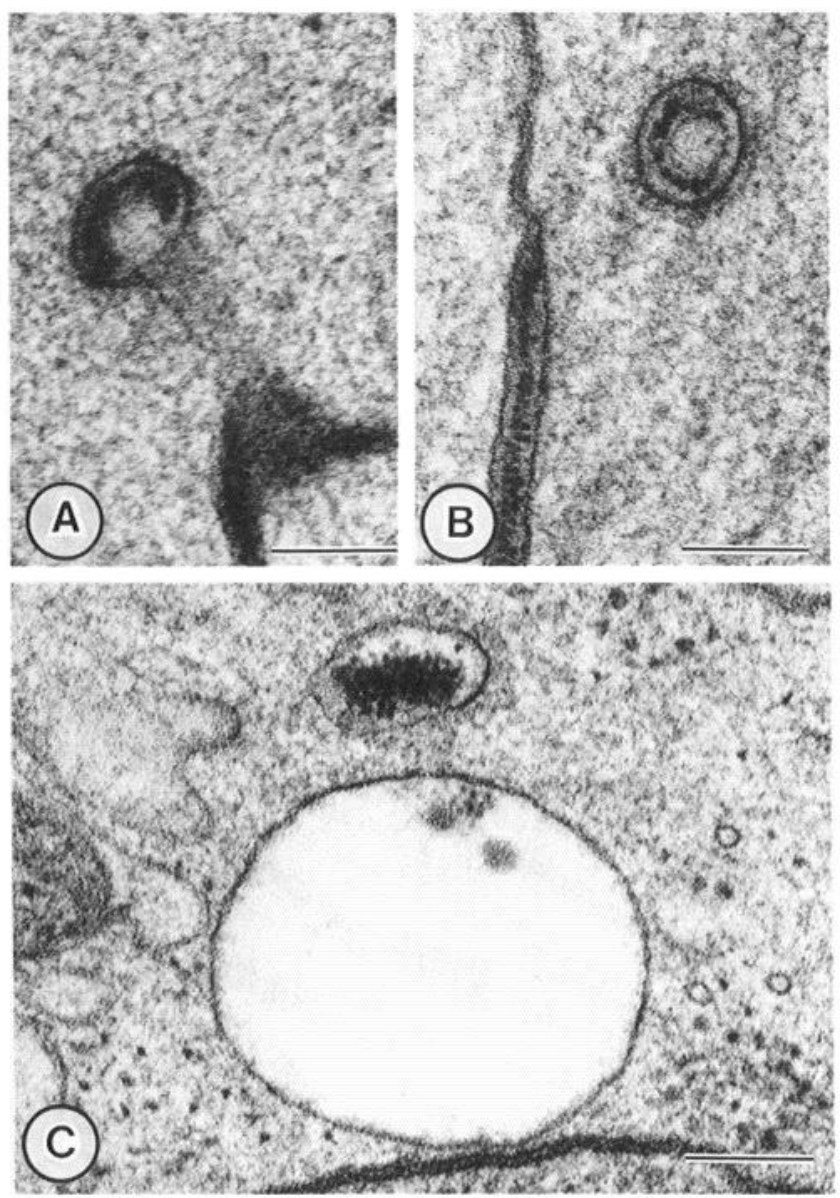

Figure 5. Sequential appearance of cationized ferritin in coated pits $(A)$, coated vesicles $(B)$, and small and large vacuoles $(C)$ at 0 (without warming to $37^{\circ} \mathrm{C}$ ), 5 , and $15 \mathrm{~min}$. This coated pit, typical of tecta frozen near $0^{\circ} \mathrm{C}$ (also see Cheng, 1986), is connected to the growth cone plasmalemma by a narrow neck, whereas the coated vesicle is not connected to the plasmalemma, as confirmed by serial sections. The ferritin particles inside the coated pit and vesicle, as well as at the surface of the growth cone plasmalemma $(B$, left), are arrayed at a fixed distance from the surface of the plasmalemma; in contrast, those inside the small and large vacuoles $(C)$ are in clusters. Scale bars, $0.1 \mu \mathrm{m}$.

substituted growth cones. Most vacuoles were spherical, but some were cup-shaped or biconcave (Fig. 1A). The lumens of most vesicles and vacuoles were clear, but some were multivesicular. Smooth vesicles were typical of the base of terminal and subterminal filopodia, but most of the spherical and, particularly, biconcave vacuoles in the growth cone were near stacks of lumenless disks (Fig. 1, $A, B$ ). An electron-dense cross-link typically bridged the spaces between vacuoles and lumenless disks (Fig. 1A, left inset) as well as the spaces between lumenless disks in the stacks.

\section{Cationized ferritin uptake}

Excised optic tecta were exposed to cationized ferritin for various times to see whether the coated pits and vesicles, uncoated vesicles and vacuoles, as well as the stacked and single lumenless disks, are functionally related. Ferritin micelles were initially $(0$ min) present in coated pits (Fig. 5A) and appeared in coated vesicles $5 \mathrm{~min}$ after the labeled tecta were warmed to $37^{\circ} \mathrm{C}$ (Fig. $5 B)$. Ferritin was found in smooth vesicles and vacuoles at longer incubation times (15-30 min; Fig. 5C); at this time, it was also occasionally present in large vacuoles, though still ab- 


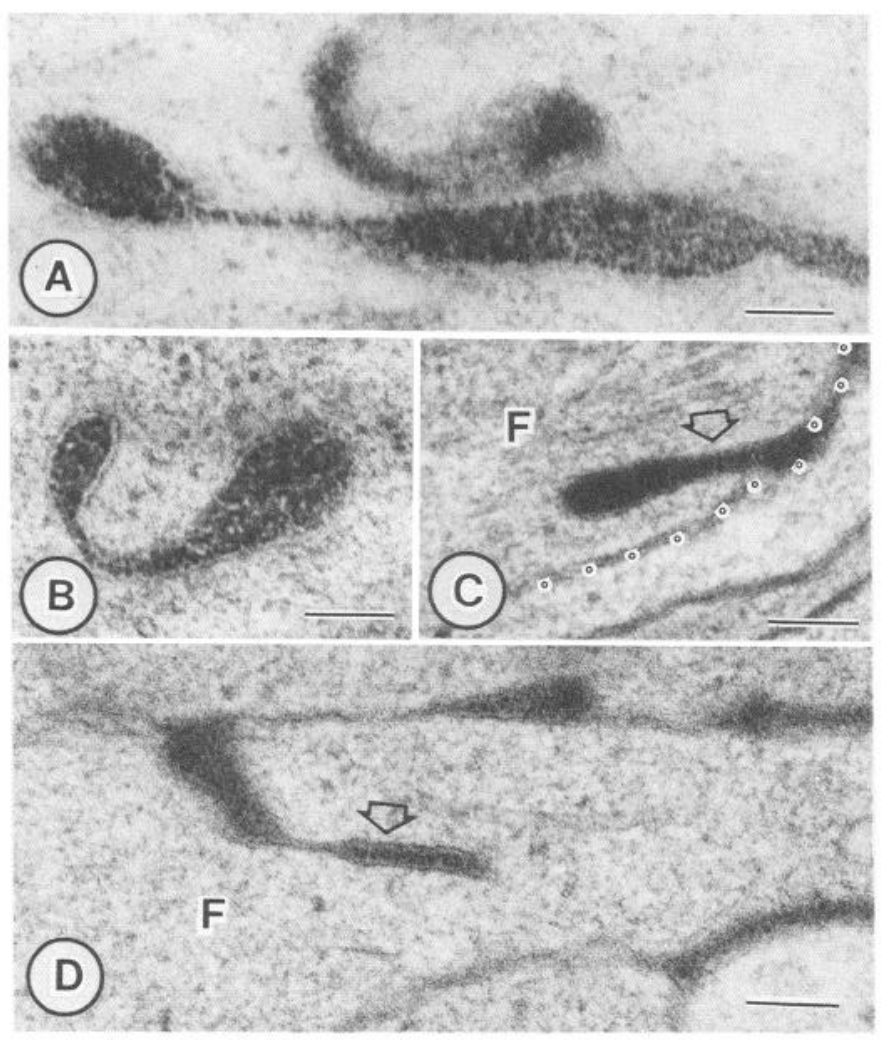

Figure 6. Cationized ferritin in stacked disks $(A)$, cup-shaped, membrane profiles $(B)$, and the disk-shaped plasmalemmal invaginations (empty arrows in $C$ and $D$ ) at $30 \mathrm{~min}$ or longer. Circle dots outline sections of the filopodium ( $F$ in $C$ and $D$ ) containing disk-shaped plasmalemmal invaginations (the outlines of obliquely sectioned plasma membranes of filopodia were determined in serial sections). Ferritin enclosed in these membrane structures is concentrated but not evenly spaced. Scale bar, $0.1 \mu \mathrm{m}$.

sent from stacked and single membrane disks. Ferritin first appeared in the stacked and single disks (Fig. 6A), as well as flat, cup-shaped profiles (Fig. $6 \mathrm{~B}$ ) and disk-shaped plasmalemmal configurations (Fig. 6, C,D) at $30 \mathrm{~min}$ of incubation.

Ferritin particles were regularly arrayed at a fixed distance from the plasmalemmal surface of the tectal growth cones, coated pits, and coated vesicles (Fig. $5, A, B$ ), suggesting specific binding of the cationized ferritin micelles to anionic sites at the plasmalemmal surface. In contrast, ferritin in the stacked and single disks was homogeneously distributed (Fig. $5 B$ ). Ferritin micelles were also uniformly distributed around the disk-shaped membrane structures contacting the growth cone plasmalemma (Fig. 6, $C, D$ ).

\section{Discussion}

Flattened membrane profiles are preserved in growth cones from chick optic tecta prepared by freeze-substitution (Cheng and Reese, 1985). These flattened membrane profiles occur individually near the base of the terminal filopodium and in stacks in the distal segments of growth cones. We now demonstrate that these profiles represent transverse sections of lumenless membrane-limited disks and that the multilamellated stacks are arrays of these disks. We also provide evidence that the single disks add membrane to the growth cone plasmalemma by exocytotic fusion and that the growth cone plasmalemma is then endocytosized by coated pits and vesicles, which, in turn, give

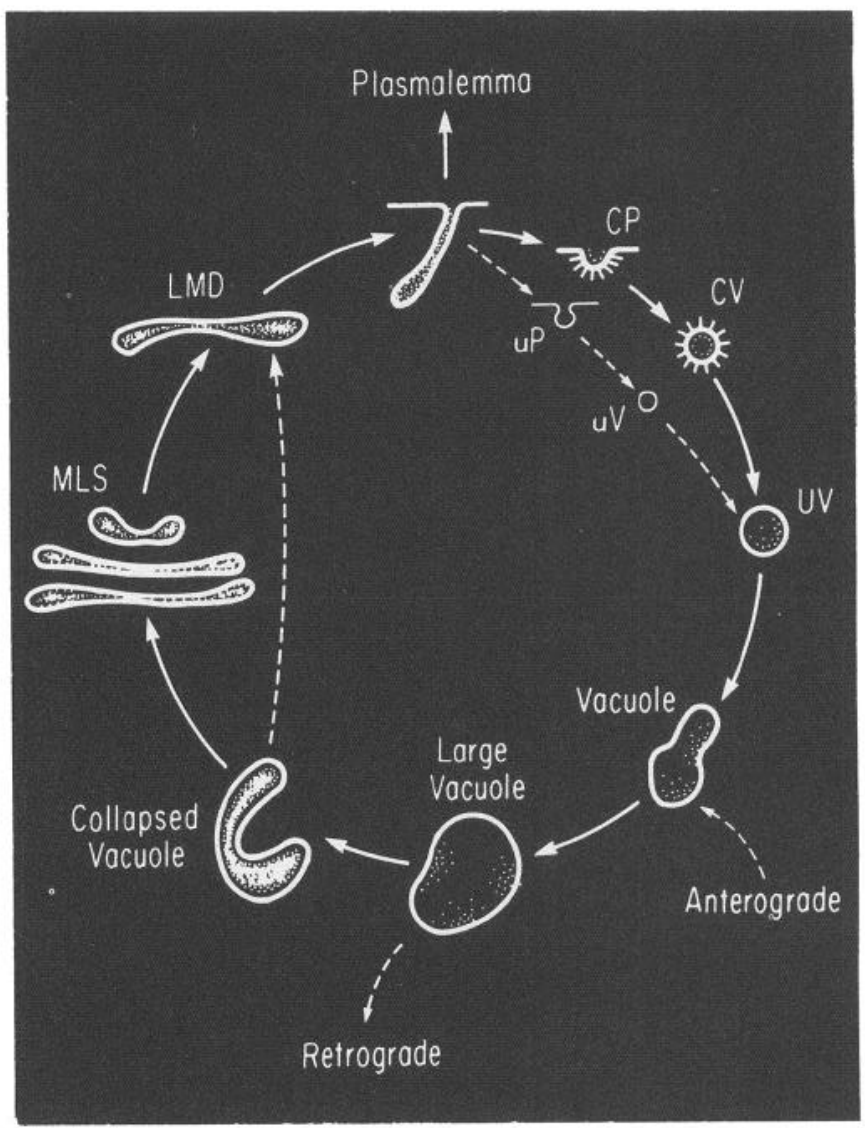

Figure 7. Hypothetical recycling of the growth cone plasmalemma. Plasma membrane is endocytosized by coated pits $(C P)$ and vesicles $(C V)$, which soon become uncoated $(U V)$. The uncoated vesicles fuse to form small and large vacuoles, which, in turn, collapse. These collapsed vacuoles may replenish the multilamellated stack $(M L S$, solid line). The multilamellated stack provides the single membrane disks ( $L M D$, dotted line), which provide membrane for exocytotic addition to the growth cone plasmalemma. Uncoated plasmalemmal pits $(u P)$ and uncoated vesicles, or vacuoles $(u V)$, could also play a role in the endocytosis of the plasmalemma (dotted lines), although this type of membrane retrieval has not been observed in the chick tectal growth cones; it is also possible that collapsed vacuoles could become single membrane disks (dotted line at left). Small-diameter membrane organelles that mediate anterograde transport, as well as large-diameter organelles that mediate retrograde transport, could be added to the cycling membrane at locations indicated by the dotted lines. Participation of smooth endoplasmic reticulum is not considered here because it never takes up ferritin. Dots inside organelles indicate ferritin.

rise to smooth vesicles and vacuoles that are interconnected to, and therefore appear to give rise to, the stacked disks. We can now postulate a membrane cycle at the growth cone tip initiated by the formation of coated pits and ending with the exocytotic addition of single membrane disks to the growth cone plasmalemma, as diagrammed in Figure 7.

\section{Endocytosis of the growth cone plasmalemma}

On the basis of ferritin uptake after short exposure times, we conclude that coated pits are the primary organelles internalizing the growth cone plasmalemma, although the uncoated pits that appear to give rise to membrane tubules (Weldon, 1975; Bunge, 1977), uncoated vesicles (Miller and Heuser, 1984), and vacuoles (Heuser et al., 1979), as well as receptosomes (Willingham and Pastan, 1980), could provide other means of internalizing 


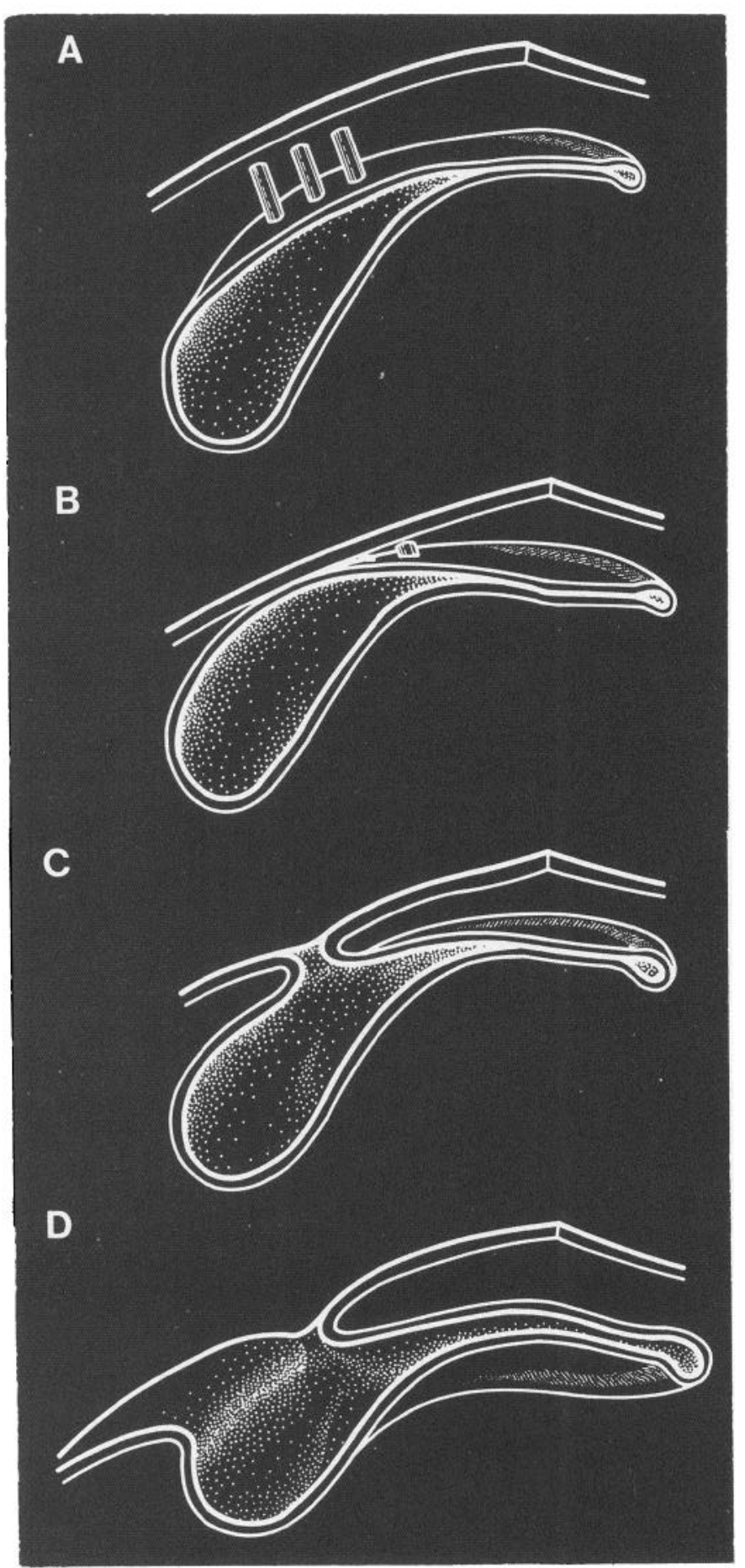

Figure 8. Postulated temporal sequence of exocytotic addition of disk membranes to the growth cone or filopodial plasmalemma. Exocytotic addition of a membrane disk to the plasmalemma is presumably initiated at a close contact between the disk and the plasmalemma $(A)$. This association, which may be stabilized by cross-bridging of fine filaments between them, leads to contact $(B)$ and eventually fusion between the 2 membrane systems $(C)$. The end result is incorporation of a lumenless membrane disk into the plasmalemma $(D)$, which could then be used for extension of a filopodium or elongation of the neurite.

plasmalemma. However, uncoated plasmalemmal pits have not been observed in the growth cones from chick optic tectum (see also Cheng and Reese, 1985).

Smooth vesicles and vacuoles are thought to derive from coated vesicles because ferritin appears in them later than in the coated vesicles. Judging from the subsequent sequential appearance of cationized ferritin in these organelles, the internalized plasmalemma is then distributed sequentially among the small and large vacuoles, cup-shaped and biconcave vacuoles, and then the stacked and single membrane disks. Endocytosis of extracellular markers by coated pits and vesicles, as well as fusions between uncoated vesicles and vacuoles, has been reported in growth cones of dissociated nerve cells (Birk et al., 1972; Weldon, 1975; Bunge, 1977), and this process of receptormediated endocytosis is well known in non-neuronal cells (Willingham and Pastan, 1980; Wall and Hubbard, 1981; Goldstein et al., 1982; van Renswoude et al., 1982; Cheng, 1986).

\section{Exocytotic addition of single disks to the plasmalemma}

Individual lumenless disks are concentrated near the base of the terminal filopodium, at the elongating end of the growth cone. These single lumenless disks are frequently located very close to and occasionally contact the plasmalemma. Fine cross-links are frequent near sites of contact between single disks and the plasmalemma, which suggest that they may hold the disks next to the plasmalemma. Similar cross-links are found at the secretory granule-plasmalemmal appositions in amebocytes, at the incipient sites of exocytosis (Ornberg and Reese, 1981). Indeed, images representative of different times in the sequential membrane approach and membrane fusion steps underlying exocytosis in freeze-substituted secretory cells have many similarities to images of disk-membrane relationships in the freezesubstituted growth cones. Secretory granules in amebocytes (Ornberg and Reese, 1981) and mast cells (Chandler and Heuser, 1980) move very close to and connect with the plasmalemma immediately before fusing with it. Some of the single disks are similarly juxtaposed to the plasmalemma of the tectal growth cones. Minute continuities between disk membrane and the filopodial plasmalemma are interpreted as narrow-necked pores, estimated to be $25 \mathrm{~nm}$ in diameter. These are similar to the narrow-necked pores $(0.05 \mu \mathrm{m}$ in diameter $)$ joining single granules and the plasmalemma in amebocytes (Ornberg and Reese, 1981) and mast cells (Chandler and Heuser, 1980). These structural similarities to secretory granule interactions with the plasmalemma during exocytotic fusion support the idea that the lumenless disks fuse with the growth cone plasmalemma. The details of the proposed exocytotic addition of disk membrane to the growth cone surface are diagrammed in Figure 8.

The structural changes reported here could, alternatively, be interpreted as indicative of direct endocytosis of the plasmalemma (Weldon, 1975; Bunge, 1977). In fact, we do not know whether the tectal growth cones were extending or retracting at the moment of freezing; endocytosis might predominate at the distal ends of growth cones retracting at the moment of freezing. However, we presume that the freeze-substituted growth cones were extending at the moment of freezing because of the consistent presence of a terminal filopodium and the similarity in the shapes of freeze-substituted tectal growth cones to the shapes of chemically fixed growth cones, which should be extending because the tectum was not cut or even touched until after fixative was perfused through the vasculature (Cheng and Reese, 1985). Also, stacks of disk-like membrane profiles are present in freeze-substituted growth cones from dissociated neurons; these growth cones were presumably growing at the moment of freezing (Rees and Reese, 1981). Moreover, with the exception 
of the single disks that were already fused with the growth cone plasmalemma at the terminal filopodium, the cationized ferritin particles do not enter the stacked disks at the earlier times when ferritin has already appeared in coated pits, coated vesicles, uncoated vesicles, and vacuoles. The packing density and distribution of the ferritin particles within stacked and single membrane disks are also similar to those within the disklike plasmalemmal configurations at the surface of the growth cone plasmalemma. Thus, there are several reasons to interpret the continuity between single disks and the plasmalemma as representive of exocytotic fusion instead of plasmalemmal endocytosis accompanying filopodial retraction. If invagination of the growth cone plasmalemma accompanies filopodial probing (Harrison, 1910; Bray, 1970; Bray et al., 1978; Kataoka et al., 1980) or tissue preparation, it does not appear to involve direct formation of disks from the plasmalemma in the chick optic tectum.

The cisternae or tubules considered part of the SER have been thought to derive directly from filopodial retraction because extracellular tracers appear in the SER before or simultaneously with their appearance in coated vesicles in cultured growth cones (Weldon, 1975; Bunge, 1977). However, much of the SER network observed in the chemically fixed growth cones from dissociated neurons (Bunge, 1973, 1977; Weldon, 1975) and intact optic tectum (Cheng and Reese, 1985) is evidently an artifact of chemical fixation because it disappears when the optic tectum is quick-frozen and freeze-substituted (Cheng and Reese, 1985). The significance of the uptake of extracellular tracers into the fixative-induced SER therefore remains unclear.

\section{Multilamellated stacks as a membrane reservoir}

Although the single lumenless disks associated with the terminal filopodia could have originated directly from vesicles and vacuoles, or even the few SER cisternae present in the freeze-substituted growth cones, they seem more likely to have derived from the multilamellated stacks, which, in turn, are derived from the vesicles and vacuoles. This conclusion is based on our observations that cationized ferritin appears first in vacuoles and later in stacked and single disks, and also that vacuoles and stacked disks are interconnected by fine, electron-dense crosslinks. Thus, the multilamellated stacks are postulated to be sites of membrane storage that give rise to the single disks that add membrane directly to the plasmalemma. Together, this internal pool of membrane represents $80 \%$ of the total intracellular membrane in the chick tectal growth cones.

\section{Local recycling of the plasmalemma}

Recycling of the plasmalemmal membrane appears to be a local activity of the growth cone because coated pits and vesicles, smooth vesicles and vacuoles, as well as the stacked and single membrane disks, are concentrated in the distal segments of the freeze-substituted growth cones from chick optic tectum (Cheng and Reese, 1985). More specifically, coated pits and vesicles are concentrated in the distal regions near the terminal filopodium (Cheng and Reese, 1985), indicating that cndocytosis of the plasmalemma occurs primarily in these regions of the tectal growth cones. In addition, single membrane disks located inside of filopodia are frequently apposed to and occasionally contact the plasmalemma, suggesting that they are added directly to the filopodial plasmalemma.

Local recycling of the growth cone plasmalemma clearly cannot account for the net gain of membrane during elongation of the axons-additional membrane from other sources would be needed for expansion of the chick tectal growth cones. One possible source of membrane is the vesicles and vacuoles that mediate microtubule-based anterograde transport (Miller and Lasek, 1985; Schnapp et al., 1985). The large vacuoles and multivesicular bodies that mediate retrograde transport (Smith, 1980) could remove membrane from the pool of membrane cycling locally in the chick tectal growth cones. A hypothetical interchange of organelles in antero- and retrograde transport with those being recycled locally is depicted in Figure 7.

\section{Role of membrane recycling}

Local membrane cycling in the chick tectal growth cones could provide a pool of membrane ready to be added to the plasmalemma. This added membrane could then be used to expand existing filopodia, to generate new filopodia, or even to add the membrane needed for neuritic outgrowth. While it is not clear whether filopodial expansion requires exocytosis of membrane from the internal pool, additional membrane is clearly needed to sustain a neuritic growth rate of $1.08 \mu \mathrm{m} / \mathrm{min}$ in the cultured neurons (Bray and Chapman, 1985). However, the additional membrane for neuritic outgrowth appears to originate from the filopodial plasmalemma, which, in turn, appears to arise from the lamellipodia at the bases of filopodia (Bray and Chapman, 1985). Thus, stacked and single disks could provide additional membrane for both filopodial and neuritic extension.

Another possible role of membrane recycling could be to recycle and therefore reutilize receptors, as yet unknown, on the plasmalemmal surface of the chick tectal growth cones. This aspect of membrane recycling in the growth cone would be similar to the recycling of cell surface receptors for low-density lipoprotein (Goldstein et al., 1982), $\alpha$-macroglobulin (Willingham and Pastan, 1980), asialoglycoprotein (Wall and Hubbard, 1981), and transferrin (van Renswoude et al., 1982; Watts, 1985; Cheng, 1986) in non-neuronal cells.

\section{References}

Birk, R. I., M. C. Mackey, and P. R. Weldon (1972) Orgnanelle formation from pinocytotic elements in neurites of cultured sympathetic ganglion. J. Neurocytol. 1: 311-340.

Bray, D. (1970) Surface movements during the growth of single explanted neurons. Proc. Natl. Acad. Sci. USA 65: 905-910.

Bray, D., and K. Chapman (1985) Analysis of microscopic movement on the neuronal growth cone. J. Neurosci. 5: 3204-3213.

Bray, D., C. Thomas, and G. Shaw (1978) Growth cone formation in cultures of sensory neurons. Proc. Natl. Acad. Sci. USA 75: 52265229.

Bunge, M. B. (1973) Fine structure of nerve fibers and growth cones of isolated sympathetic neurons in culture. J. Cell Biol. 56: 713-735.

Bunge, M. B. (1977) Initial endocytosis of peroxidase or ferritin by growth cones of cultured nerve cells. J. Neurocytol. 6: 407-439.

Chandler, D. E., and J. E. Heuser (1980) Arrest of membrane fusion events in mast cells by quick-freezing. J. Cell Biol. 86: 666-674.

Cheng, T. P. O. (1986) Redistribution of cell surface transferritin receptors prior to their concentration in coated pits as revealed by immunoferritin labelling. Cell Tissue Kes. 244: 613-619.

Cheng, T. P. O., and T. S. Reese (1985) Polarized compartmentalization of organelles in growth cones from developing optic tectum. J. Cell Biol. 101: 1473-1480.

Feldman, E. L., D. Axelrod, M. Schwartz, A. M. Heacock, and B. W. Agranoff (1981) Studies on the localization of newly added membranes in growing neurites. J. Neurobiol. 12: 591-598.

Goldstein, J. L., M. S. Brown, R. G. W. Anderson, and S. K. Basu (1982) Recycling of cell surface receptors: Observations from the LDL system. Cold Spring Harbor Symp. Quant. Biol. 46: 713-722.

Harrison, R. G. (1910) The outgrowth of the nerve fiber as a mode of protoplasmic movement. J. Exp. Zool. 9: 787-84G. 
Heuser, J. E., T. S. Reese, M. J. Dennis, Y. Jan, L. Jan, and L. Evans (1979) Synaptic vesicle exocytosis captured by quick freezing and correlated with quantal transmitter release. J. Cell Biol. 81: 275-300.

Kataoka, S., D. Sandquist, L. Williams, and T. H. Williams (1980) Growth cones in differentiated neuroblastoma: A time-lapse cinematographic and electron microscopic study. J. Neurocytol. 9: 591602.

Kawana, E., C. Sandri, and K. Akert (197I) Ultrastructure of growth cones in the cerebellar cortex of the neonatal rat and cat. Z. Zellforsch. Mikrosk. Anat. 115: 284-298.

Landis, S. C. (1978) Growth cones of cultured sympathetic neurons contain adrenergic vesicles. J. Cell Biol. 78: R8-14.

Miller, R. T., and R. J. Lasek (1985) Cross-bridges mediate anterograde and retrograde vesicle transport along microtubules in squid axoplasm. J. Cell Biol. 101: 2181-2193.

Miller, T. M., and J. E. Heuser (1984) Endocytosis of synaptic vesicle membrane at the frog neuromuscular junction. J. Cell Biol. 98: 685689.

Nordlander, R. H., and M. Singer (1982) Morphology and position of growth cones in the developing Xenopus spinal cord. Dev. Brain Res. 4: 181-193.

Ornberg, R. L., and T. S. Reese (1981) Beginning of exocytosis captured by rapid-freezing of Limulus amebocytes. J. Cell Biol. 90:4054.

Pfenninger, K. H., and M. Maylie-Pfenninger (1981) Lectin labeling of sprouting neurons: I. Regional distribution of surface glycoconjugates. J. Cell Biol. 89: 536-546.

Rees, R. P., and T. S. Reese (1981) New structural feature of freezesubstituted neuritic growth cones. Neuroscience 6: 247-254.

Schnapp, B. J., R. D. Vale, M. P. Sheetz, and T. S. Reese (1985) Single microtubules from squid axoplasm support bidirectional movement of organelles. Cell 40:455-462.

Smith, R. S. (1980) The short term accumulation of axonally-transported organelles in the region of localized lesions of single myelinated axons. J. Neurocytol. 9: 39-65.

Speidel, C. C. (1933) Studies of living nerve. II: Activities of amoeboid growth cones, sheath cells and myelin segments as revealed by prolonged observation of individual nerve fibers in frog tadpoles. Am. J. Anat. 52: 1-75.

Tennyson, V. M. (1970) The fine structure of the axon and growth cone of the dorsal root neuroblast of the rabbit embryo. J. Cell Biol. 44: $62-79$.

van Renswoude, J., K. R. Bridges, J. B. Harford, and R. D. Klausner (1982) Receptor-mediated endocytosis of transferrin and the uptake of $\mathrm{Fe}$ in $\mathrm{K} 562$ cells: Identification of a non-lysosomal acidic compartment. Proc. Natl. Acad. Sci. US $\Lambda$ 79: 6186-6190.

Wall, D. A., and A. L. Hubbard (1981) Galactose-specific recognition system of mammalian liver: Receptor distribution on the heptocyte cell surface. J. Cell Biol. 90: 687-696.

Watts, C. (1985) Rapid endocytosis of the transferrin receptor in the absence of bound transferrin. J. Cell Biol. 100: 633-637.

Weldon, P. K. (1975) Pinocytotic uptake and intracellular distribution of colloidal thorium dioxide by cultured sensory neurites. J. Neurocytol. 4: 341-356.

Willingham, M. C., and I. Pastan (1980) The receptosome: An intermediate organelle of receptor-mediated endocytosis in cultured fibroblast. Cell 21: 67-77.

Yamada, K. M., B. S. Spooner, and N. K. Wessels (1971) Ultrastructural and function of growth cones and axons of cultured nerve cells. J. Cell Biol. 49: 614-635. 